\title{
Modified Ponseti Technique in an Eleven-Year-Old with Bilateral Untreated Clubfoot: A Case Report
}

\author{
Maria Wong (iD) 1,2 \\ Mazelan Ali \\ Aik Saw ${ }^{1,2}$ \\ 'Department of Orthopaedic Surgery, \\ University Malaya Medical Centre, Kuala \\ Lumpur, Malaysia; ${ }^{2}$ National Orthopaedic \\ Center of Excellence for Research \& \\ Learning (NOCERAL), University of \\ Malaya, Kuala Lumpur, Malaysia
}

\begin{abstract}
We report the case of an eleven-year-old girl with untreated bilateral clubfoot. She had a total of nine long leg castings changed weekly, Achilles tendon Z-lengthening and posterior ankle release followed by another six weeks of long leg cast immobilization postoperatively. We encouraged using night-time foot abduction brace for twelve months. Independent walking was achieved six months post-treatment. No recurrence was noted at eighteen months following treatment. The modified Ponseti weekly casting method was successful up to adolescent age; however, more cast changes, longer stretching before each cast application and posterior ankle release will be needed to achieve functional correction.
\end{abstract} Keywords: Ponseti treatment, neglected clubfoot, untreated clubfoot

\section{Introduction}

The Ponseti method has been shown to be effective in treating clubfoot of all etiologies, however the upper age limit for this treatment has not been defined. Walking-age children with clubfoot deformities managed with Ponseti serial casting have been increasingly reported in the literature over the past decade. To the best of our knowledge, the oldest reported case was a nineteen-year-old from Iran. We demonstrate our detailed method of Ponseti casting in an 11-year-old with untreated clubfeet.

\section{Statement of Informed Consent}

The family agreed and consented to the images and medical information being submitted for publication. Written consent was given by the guardians. Institutional approval for the case details was not required, as anonymity was protected.

\section{Case Report}

A healthy eleven-year-old female with bilateral clubfoot was referred to our center by a charity outreach organization. She was from a remote area in Kalimantan, Borneo, and her childhood was handicapped by this deformity. Clinically, both her feet were grossly deformed and stiff with forefoot adduction, midfoot cavus, hindfoot varus and rigid equinus (Figure 1). She had adapted to walking short distances on the talar heads and the dorsum of her forefoot, resulting in callus formation over the dorsum of both talar prominences (Figure 2A and B). This was a limitation for her as she was unable to attend school regularly and was homebound.

Weekly changes of nine long leg casts were done in our dedicated outpatient clubfoot plaster room. We used a bolster under the popliteal fossa. An inner cotton stockinette and soft band was applied beyond the toes then up until midthigh.
Correspondence: Maria Wong Department of Orthopaedic Surgery, University Malaya Medical Centre, Kuala Lumpur, 50603, Malaysia

Tel +6037949206

Fax +60379493|4

Emailmria_lynn@yahoo.com 


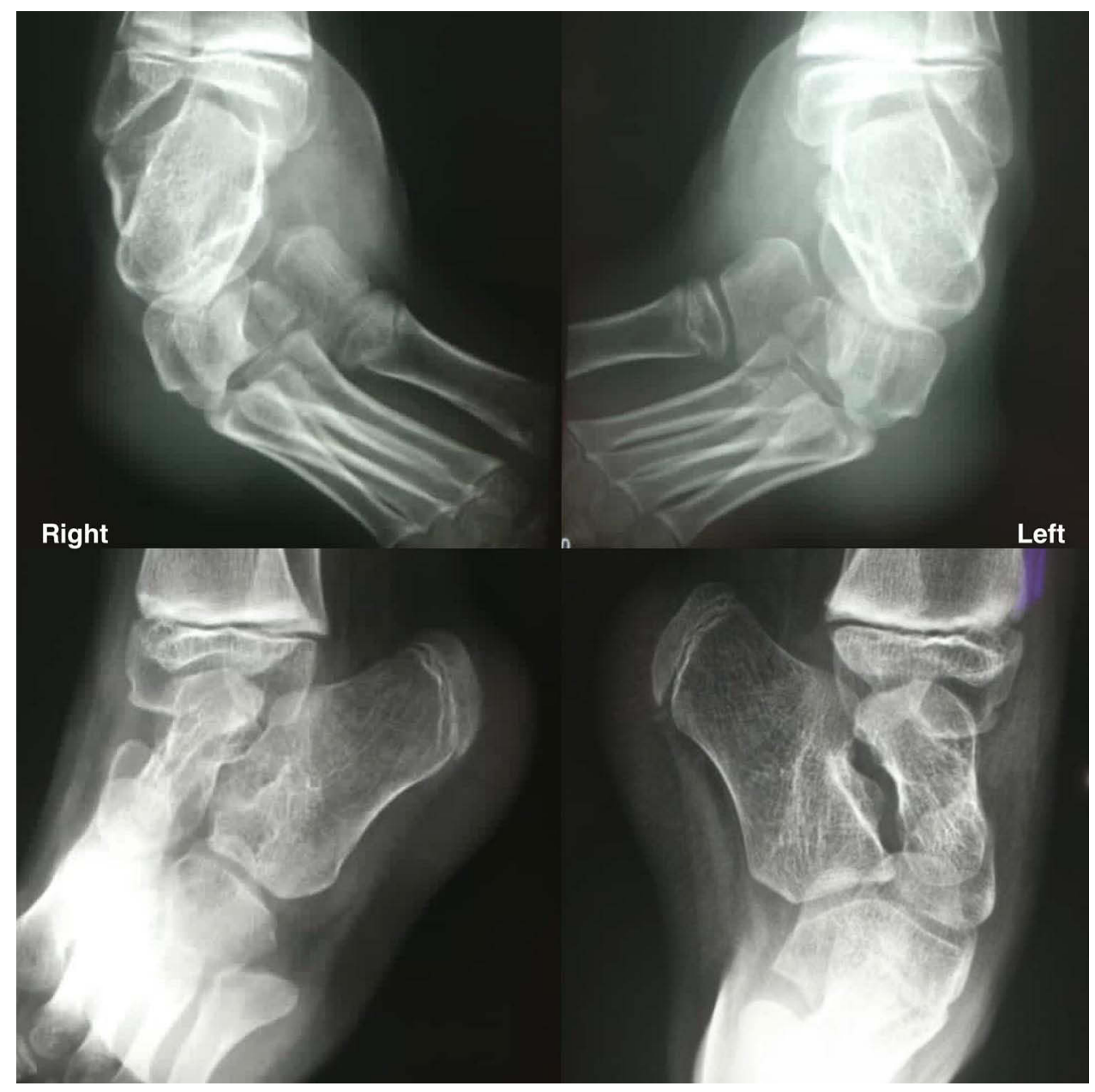

Figure I The patient's plain radiographs before treatment.
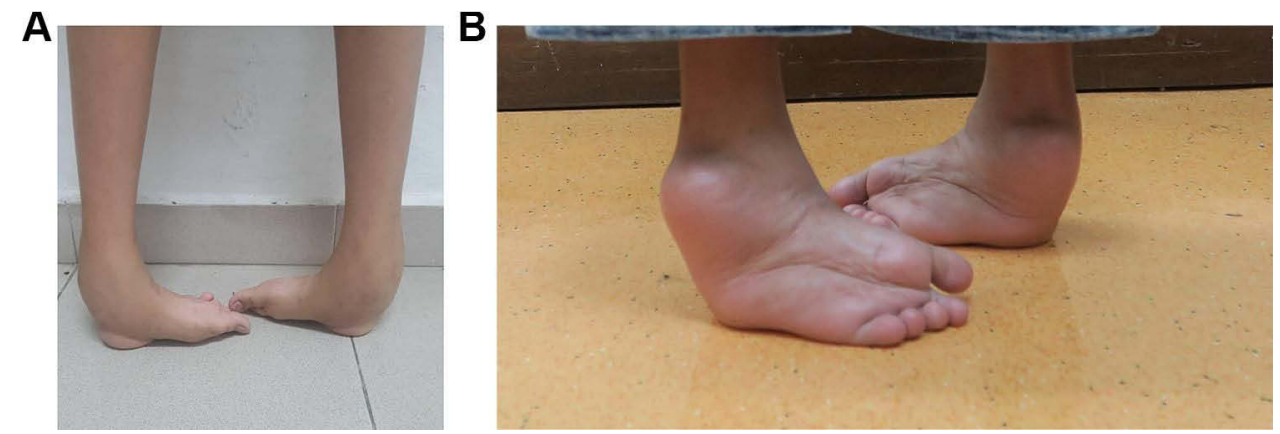

Figure 2 (A) Bilateral untreated clubfoot seen anteriorly; weightbearing on talus prominences and dorsum of the forefeet. (B) The same clubfoot seen from posterior in standing position; both feet adducted and supinated with equinus deformity.

Sustained manual stretching of her feet was done for five minutes with twenty seconds rest in-between stretches before applying the cast in maximum stretch. This was done before every casting, and her discomfort gradually increased when we started stretching her feet into abduction. The discomfort was temporary, occurring only during the stretching and she was comfortable after the cast had set. The foot correction was held by one person; one hand grasping the forefoot under the stockinette, the other using thenar eminence over the talar prominence (this pressure was relieved intermittently to prevent skin necrosis), while avoiding the calcaneum to allow free rotation under the 
talus. Plaster of Paris was then applied over the foot to midleg, moulding was done in the corrected foot position of the week, the distal stockinette was then pulled over just until the tips of toes were seen, and the cast was allowed to set. The foot and distal tibia were then planted on the bed and positioned in external rotation with an assistant stabilizing the knee; the bolster was removed here, and the Plaster of Paris was completed to midthigh with the knee in $45^{\circ}$ flexion. This method of keeping the heel static on the bed holds the knee in constant flexion and allows smooth cast application without creasing the popliteal area. Following this, a roll of 4-inch fiberglass cast was applied for cast longevity, as we expected her to move for wheelchair transfers, and bottom shuffling on the floor (Figures 3 and 4).

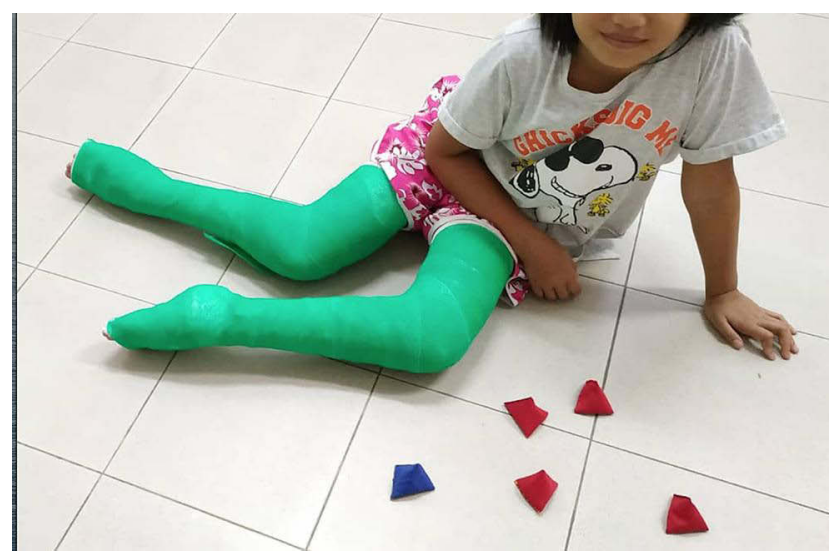

Figure 3 Sitting on the floor in long leg casts; she was able to move across the room despite the casts.

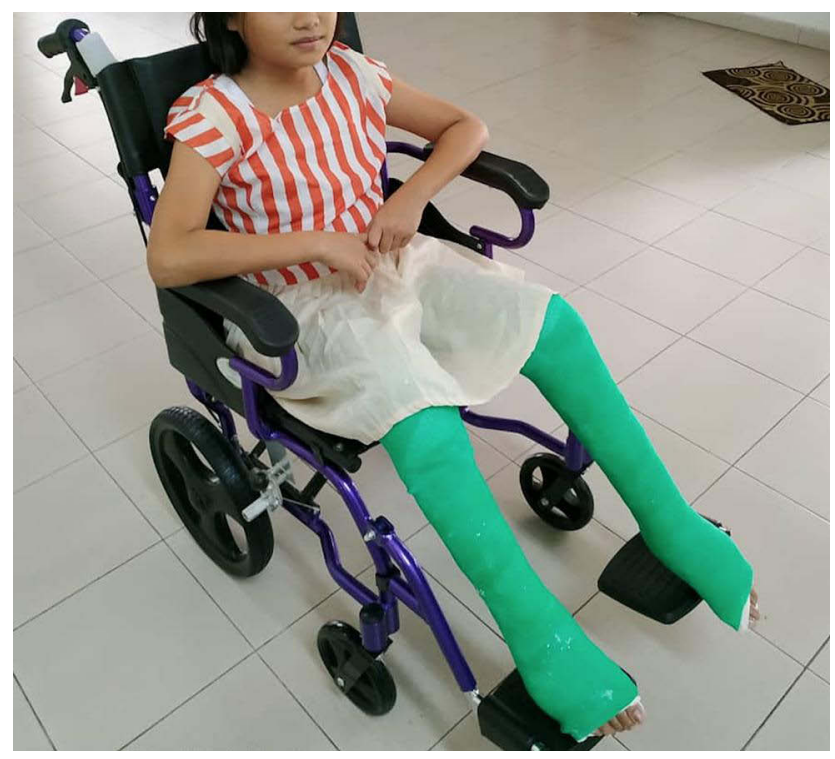

Figure 4 Long-leg bent-knee cast was convenient for wheelchair mobilization and car rides.
This outer water-resistant layer was added to the original Ponseti cast to cater to local and cultural setting.

Cast 1 and 2 were aimed at midfoot cavus correction with the forefeet casted in supination to align the hindfoot with the forefoot ( 2 weeks). We started forefoot abduction at cast 3 through cast 9 ( 7 weeks). We found we could achieve only few degrees of abduction within the patient's tolerance each week and the best final abduction at week 9 was at $30^{\circ}$ with the head of talus satisfactorily covered and the heels in neutral (Figure 5).

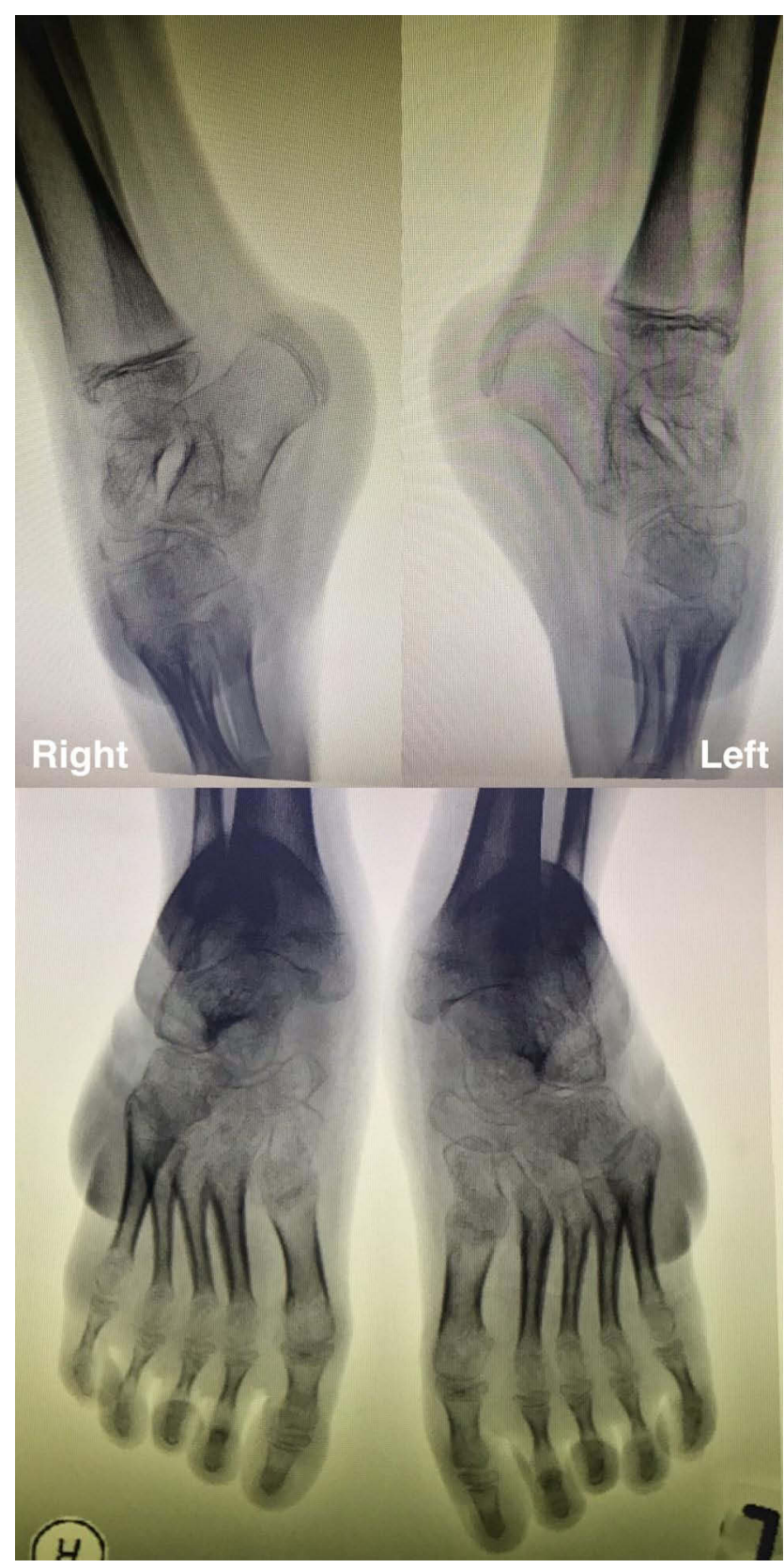

Figure 5 Repeat radiographs prior to posterior ankle release showing improved forefoot abduction. 
This achievement in abduction is notably less than the original Ponseti method in an infant. The equinus deformity was rigid and we were unable to manually stretch the Achilles tendon. She had a Z-lengthening of both Achilles tendons with posterior tibio-talar and subtalar capsulotomy to achieve $15^{\circ}$ dorsiflexion and was then immobilized in these knee casts for six weeks to allow healing of the Achilles tendon (with wound checks at three weeks and re-casting) (Figure 6).

We applied foot abduction brace (Figure 7) for night-time and recommended regular shoes in the daytime. She had painless range with $10^{\circ}$ dorsiflexion, $45^{\circ}$ plantar flexion with $20^{\circ}$ inversion and $10^{\circ}$ eversion at



Figure 6 Alignment of the feet at the conclusion of casting showing marked improvement and dried callosity over the dorsolateral aspect.

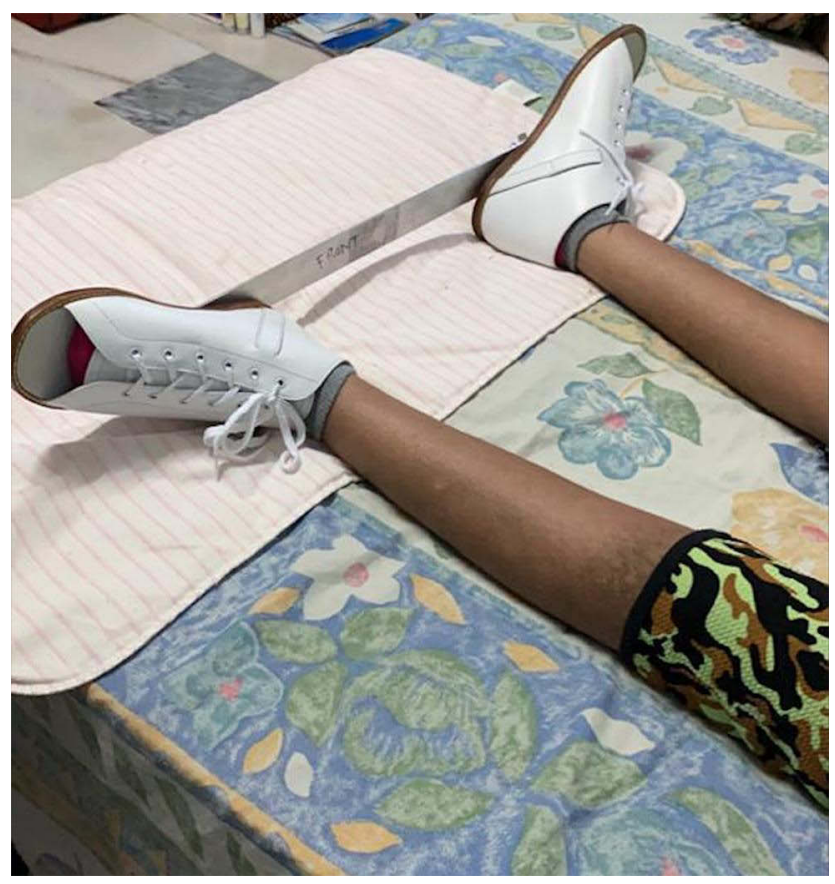

Figure 7 Foot abduction brace.

the conclusion of casting. We lost her to physical follow-up following her return to her country. By electronic communication with her carers, we discovered she was able to walk to school on uneven terrain (pebbled, mud path) six months after seeing us, and even participated in her school dance performance six months thereafter (Figures 8 and 9). At eighteen months post-treatment, she had picked up bicycle riding and was walking barefoot with no discomfort or pain in her feet and ankles, despite her radiographs (Figure 10).

\section{Discussion}

The Ponseti method consists of serial manipulation and long leg casting, tenotomy of the Achilles tendon and foot abduction brace. ${ }^{1}$ A plantigrade foot with good motion, painless and normal function is considered a successful outcome. A recent systematic review examined the efficacy of Ponseti method in older children and concluded that Ponseti should still be the first line of treatment irrespective of the deformity, and that the upper age limit for successful outcome remains uncertain. $^{2,3}$ When left untreated, clubfoot is a functional and esthetic problem which affects mobility and quality of life. Neglected clubfoot 


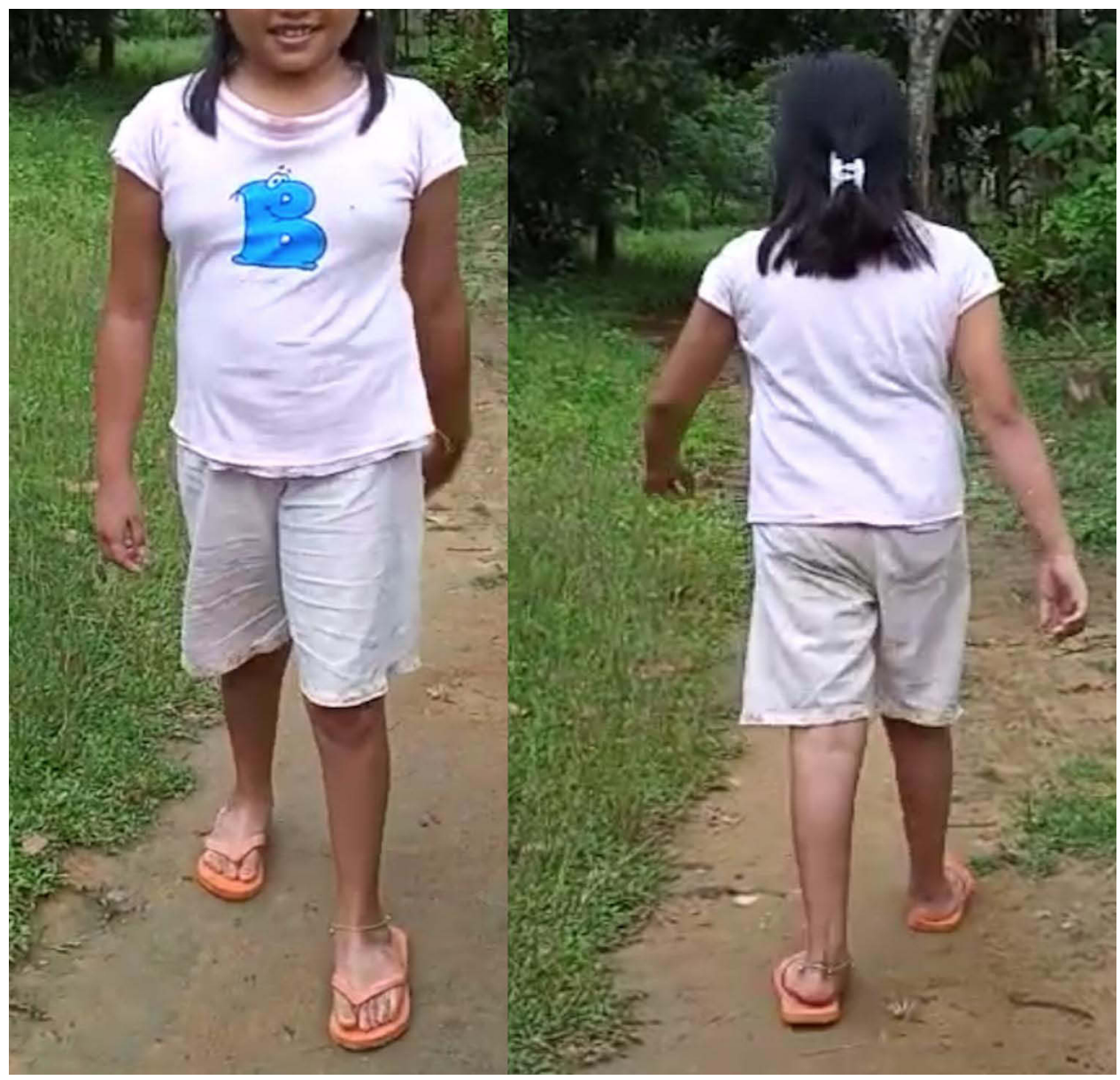

Figure 8 Twelve months post Ponseti treatment with independent walking on uneven earth terrain.

in older children is also more challenging to treat as the foot is less elastic. By definition, a child with clubfoot without treatment within the first two years of life is considered to have "neglected clubfoot". 4

Our patient was eleven years old and her feet were stiff and took longer to achieve abduction to $30^{\circ}$ at best, and the equinus deformity was rigid toward the end of casting. We applied long-leg bent-knee casts to provide better stretch and to prevent ankle/talus rotation; a total of nine casts in nine weeks. This was done in our clubfoot clinic without sedation or analgesia; in retrospect, the number of casts could have been reduced if done under anesthesia but this would have involved admission and procedural costs. Lourenco and Morcuende (oldest patient: nine years) and Ayana et $\mathrm{al}^{6}$ (oldest patient: ten years) also applied long leg casts but with two weekly intervals; resulting in doubled cast duration.
Our patient's time in casts was reduced in comparison and we found one week was adequate in-between changes as the casts were soft at the heels and knees by next review.

We found similar increased time (minimum 5 minutes) to perform the stretches prior to the cast application. ${ }^{5,7}$ Our patient's feet were stiffer than our usual infant clubfoot and we could only achieve $30^{\circ}$ of abduction, in keeping with available reports. ${ }^{5,6}$

Most reports agreed that in older walking children, additional posterior release (tibio-talar and subtalar capsulotomy) needs to be done to correct equinus deformity. ${ }^{4-6}$ We found this to be true in our elevenyear-old, her posterior contracture required Z-lengthening of tendoachilles, tibio-talar and subtalar capsulotomies. In 2016, Bashi et $\mathrm{al}^{7}$ of Iran, reported their oldest at 19 years old, also required plantar fascia 

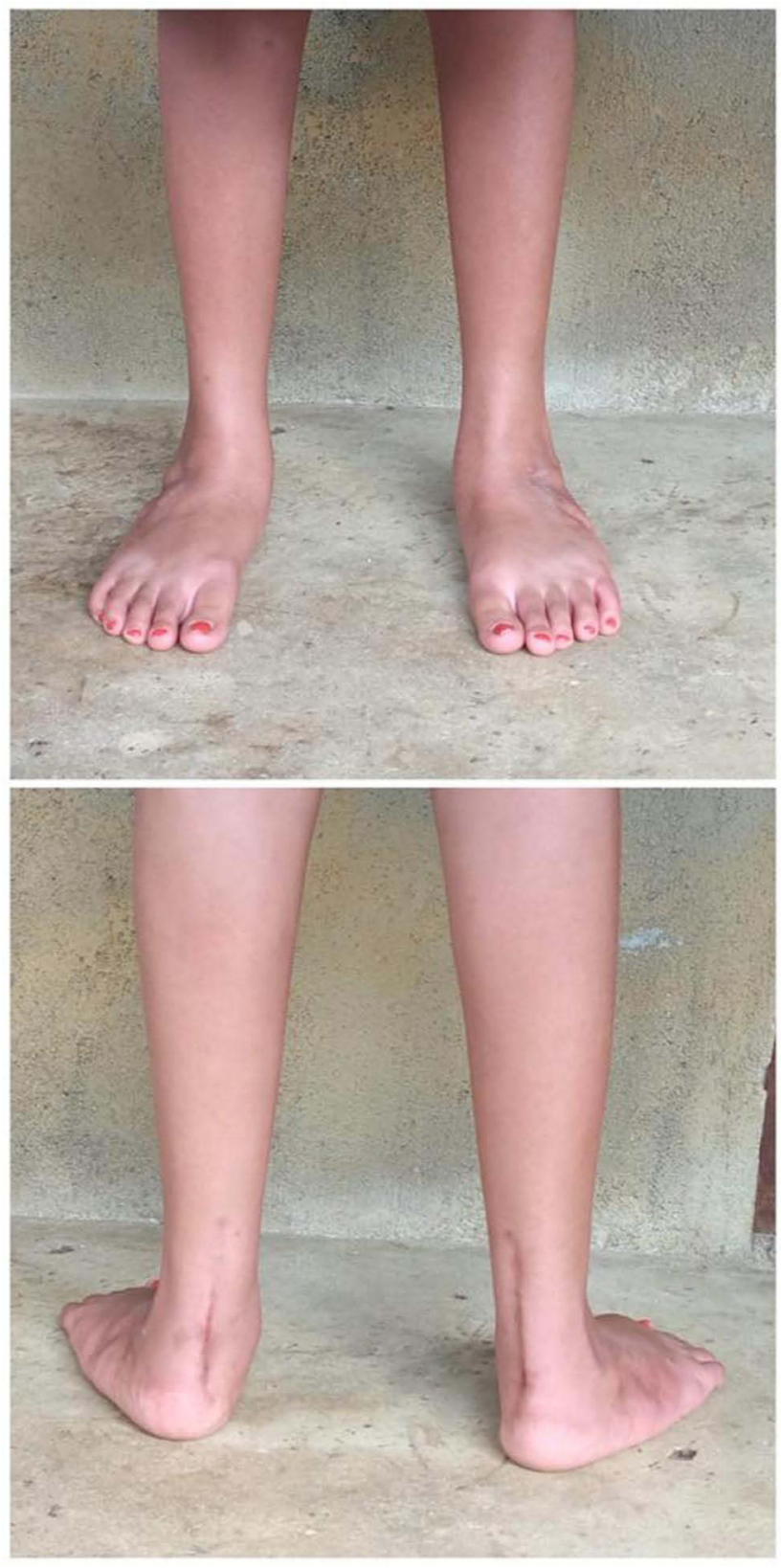

Figure 9 Near normal looking feet and no recurrence of clubfoot at twelve months; previous callosity had resolved with neutral to mild valgus heels.

release and posterior tibio-talar capsulotomy. We did not consider performing a prophylactic tibialis anterior tendon transfer at the time as the residual deformity was mainly equinus. This procedure was recommended to prevent relapses especially in children older than four years from distant regions with the risk of follow-up loss. ${ }^{3}$ Our patient did not have recurrence eighteen months later and she was functioning well with painless feet.

In contrast to most reports, ${ }^{5-7}$ we decided to use the foot abduction brace (FAB) instead of ankle foot orthosis (AFO). This is to maintain her feet corrected to $15^{\circ}$ dorsiflexion and abduction $30^{\circ}$. We were not convinced that the AFO would be able to maintain the foot abduction component effectively without the bar inbetween. We attempted to encourage the FAB for twelve months ${ }^{4,5}$ as this was the common duration for AFO in previous reports, however we lost her from physical follow-up with the start of the COVID-19 pandemic and international border lockdown. We are, therefore, unable to comment on the maintenance regime other than verbal reports of good compliance from her carers. We were relieved to observe correction was maintained from her pictures and videos sent to us a year later.

We still lack information with regards to the best maintenance protocol following Ponseti serial casting in adolescent children whose gait had been abnormal up until the point of intervention. Available literature reported satisfactory outcomes with low recurrence rate in neglected clubfeet treated with the Ponseti method after walking age. ${ }^{8}$ We decided to use the FAB for twelve months at night with normal shoes for daytime. We allowed only night-time use as our patient is weight bearing in the daytime and this axial loading would help in maintaining her plantigrade feet. We were not certain of clubfoot relapse risks in walking children at this age and whether they require similar protocol as per Ponseti. Based on our experience with other equinus deformities, there may be a risk of recurrence without night splinting and stretching exercises, hence it is reasonable to advise continued bracing.

\section{Conclusion}

Serial weekly Ponseti casting is still effective even in adolescent children and older, however, treatment may include more cast changes, followed by extensive posterior release of the ankle to correct equinus. 


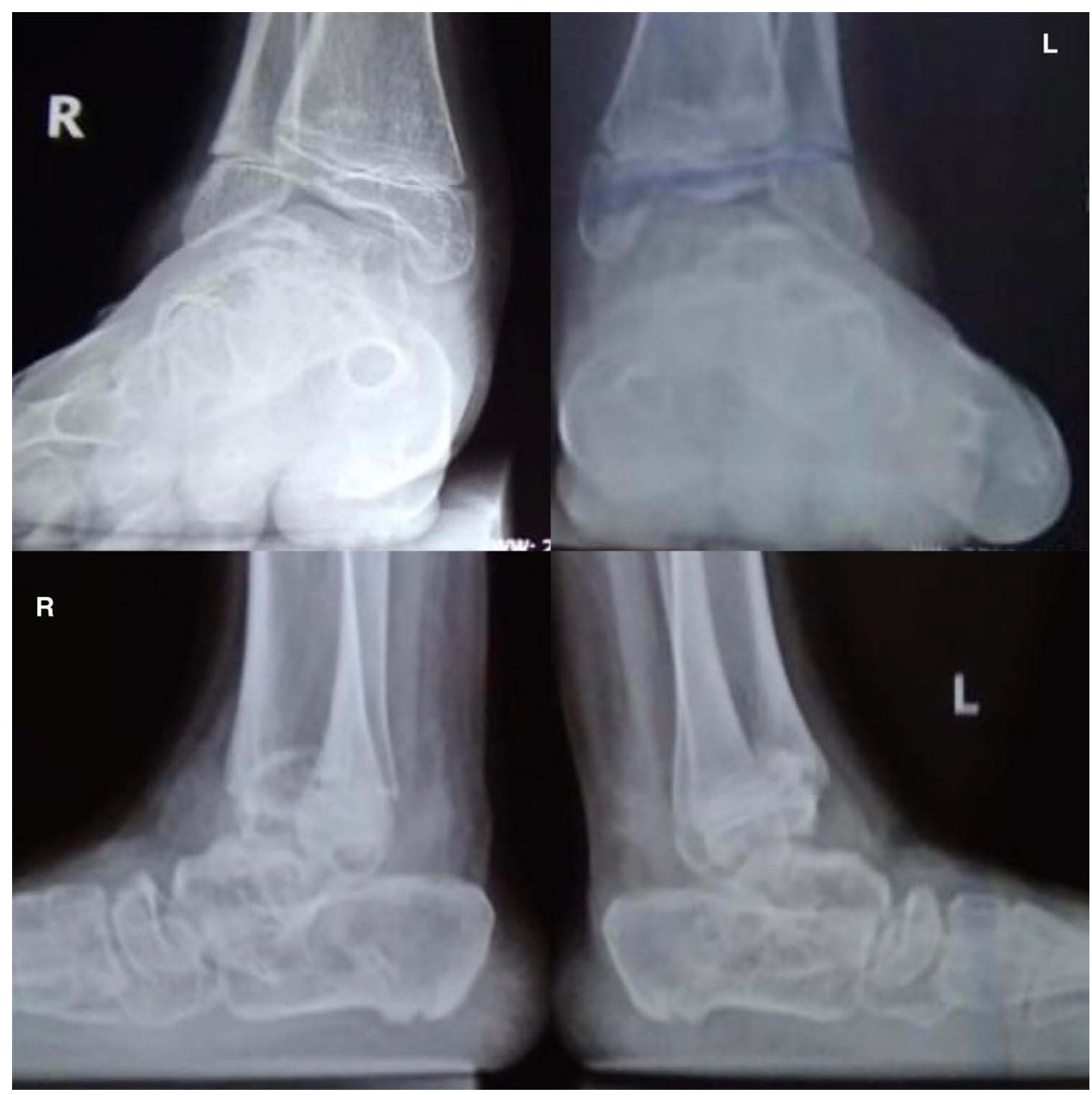

Figure 10 Weight-bearing views of both ankles at eighteen months with good function and painless ambulation.

\section{Disclosure}

The authors report no conflicts of interest in this work.

\section{References}

1. Dobbs MB, Gurnett CA. Update on clubfoot: etiology and treatment. Clin Orthop Relat Res. 2009;467(5):1146-1153. doi:10.1007/s11999009-0734-9

2. Digge V, Desai J, Das S. Expanded Age indication for Ponseti method for correction of congenital idiopathic talipes equinovarus: a systematic review. J Foot Ankle Surgery. 2018;57(1):155-158. doi:10.1053/j.jfas.2017.08.015

3. de Podesta Haje D, Maranho DA, Ferreira GF, et al. Ponseti method after walking age - a multi-centric study of 429 feet: results, possible treatment modifications and outcomes according to age groups. Iowa Orthop J. 2020;40(2):1-12.
4. Sengupta A. The management of congenital talipes equinovarus in developing countries. Int Orthop. 1987;11(11):183-187. doi:10.1007/ BF00271445

5. Lourenço AF, Morcuende JA. Correction of neglected idiopathic club foot by the Ponseti method. J Bone Joint Surg Br. 2007;89 (3):378-381. doi:10.1302/0301-620x.89b3.18313

6. Ayana B, Klungsøyr PJ. Good results after Ponseti treatment for neglected congenital clubfoot in Ethiopia. A prospective study of 22 children ( 32 feet) from 2 to 10 years of age. Acta Orthop. 2014;85(6):641-645. doi:10.3109/17453674.2014.957085

7. Bashi RH, Baghdadi T, Shirazi MR, Abdi R, Aslani H. Modified Ponseti method of treatment for correction of neglected clubfoot in older children and adolescents-a preliminary report. J Pediatr Orthop B. 2016;25(2):99-103. doi:10.1097/bpb.0000000000000266

8. Ferreira GF, Stéfani KC, Haje DP, Nogueira MP. The Ponseti method in children with clubfoot after walking age - Systematic review and metanalysis of observational studies. PLoS One. 2018;13(11): e0207153. doi:10.1371/journal.pone.0207153 


\section{Publish your work in this journal}

Orthopedic Research and Reviews is an international, peer-reviewed, open access journal that focusing on the patho-physiology of the musculoskeletal system, trauma, surgery and other corrective interventions to restore mobility and function. Advances in new technologies, materials, techniques and pharmacological agents are particularly

Submit your manuscript here: https://www.dovepress.com/orthopedic-research-and-reviews-journa welcome. The manuscript management system is completely online and includes a very quick and fair peer-review system, which is all easy to use. Visit http://www.dovepress.com/testimonials.php to read real quotes from published authors. 